\title{
Tangence
}

\section{Clivage relationnel et violence}

Éléments pour une théorie politique de la relation. À partir de

la pensée de Frantz Fanon

\section{Jean-Ernest Joos}

Numéro 63, juin 2000

Fictions et politique

URI : https://id.erudit.org/iderudit/008184ar

DOI : https://doi.org/10.7202/008184ar

Aller au sommaire du numéro

Éditeur(s)

Presses de l'Université du Québec

ISSN

0226-9554 (imprimé)

1710-0305 (numérique)

Découvrir la revue

Citer cet article

Joos, J.-E. (2000). Clivage relationnel et violence : éléments pour une théorie politique de la relation. À partir de la pensée de Frantz Fanon. Tangence, (63), 96-108. https://doi.org/10.7202/008184ar d'utilisation que vous pouvez consulter en ligne.

https://apropos.erudit.org/fr/usagers/politique-dutilisation/ 


\section{Clivage relationnel et violence. Éléments pour une théorie politique de la relation. À partir de la pensée de Frantz Fanon Jean-Ernest Joos, Collège Marie-de-France}

Nous voudrions poser la question de la violence dans la cadre d'une théorie politique de la relation. Identifier une violence comme étant relationnelle semble bien être une tautologie. Toute violence, réelle ou symbolique, réelle et symbolique, vient toujours de l'Autre. Pourtant, proposer une réflexion sur la violence dans les relations implique un choix épistémologique. Il s'agit de se demander si nous pouvons localiser une violence au sein même des relations, dans un entre-deux, tout en mettant entre parenthèses la question de l'origine de la violence et celle du sujet qui la subit. Pouvons-nous identifier ainsi des relations qui sont, par leur structure même, violentes? La question n'est bien sûr pas nouvelle, mais nous voudrions la poser à la limite du paradoxe, là où la violence se retourne contre la relation ellemême. Une relation violente en est une qui, au lieu de mettre le sujet en relation, viendrait limiter ses possibilités de relation à l'altérité. Nous proposons ici un concept unique, instrumental, aux fonctions multiples, pour comprendre comment une relation peut se mettre en place en imposant au sujet une limitation violente dans ses possibilités identitaires. Il s'agit du concept de relation clivée.

Ancré dans le vocabulaire psychanalytique, le concept fait appel pourtant à une articulation nouvelle du singulier et du collectif, où aucune de ces deux dimensions ne seraient données comme identité, mais n'existeraient que dans le rapport qui les unit. Freud fait intervenir le concept de clivage dans le cadre de ses réflexions sur la perversion et la psychose. Le sujet clivé est celui qui va soutenir conjointement et sans dialectique deux rapports inconciliables à la réalité, dont l'un relève soit du déni, soit du délire. Or, dans certaines situations de violence symbolique, le sujet se clive au sens freudien du terme. L'exemple le plus beau parce que le plus évident, imaginé par Toni Morrison dans The 
Bluest Eye ${ }^{1}$, est celui de la petite fille noire qui rêve d'avoir les yeux bleus, et qui enfin les aura. Son moi se dédouble alors et son image dans le miroir en vient à dialoguer avec une image délirante de soi, la petite fille noire aux yeux bleus. C'est pourtant, face à ces exemples d'hallucination dont parle aussi Frantz Fanon, que vient la nécessité de briser les limites de la psychanalyse et de faire intervenir un clivage de la relation. En effet, nous ne saurions rendre compte d'un clivage politiquement imposé, en l'inscrivant simplement dans la genèse d'un sujet. Une relation clivée placerait plutôt le sujet entre des possibilités relationnelles incompatibles, mais imposées, le forçant à se cliver pour se maintenir en relation. Dans ce hiatus entre l'identité et la relation, il y a l'espace de la violence, mais aussi de la résistance d'un sujet qui ne peut jamais être "tout à fait " identifié à ce que la situation relationnelle a produit comme effet sur lui. Par cette approche très locale et ponctuelle du politique, nous n'enfermons aucune victime dans un destin ou une impuissance, nous laissons plutôt aux agents la possibilité de créer leur propre pouvoir. Le déplacement de l'identité vers la relation est alors un geste politique essentiel, puisqu'il permet d'identifier la violence subjective subie comme étant à la fois interne et externe. Il ne s'agit pas seulement de poser la cause de la violence hors du sujet, mais bien aussi son effet. Une violence relationnelle est celle qui affecte le sujet dans son rapport au monde, défigurant son image de soi, restreignant ses possibilités d'identification et même d'action. Et, pour des raisons politiques essentielles, le travail de Toni Morrison, à la suite de son premier roman, va s'orienter de plus en plus vers une réflexion relationnelle, jusqu'à la théorie de Beloved et au tableau des multiples relations engendrées par la ségrégation, l'esclavage et les formes de résistance.

C'est en 1952 que nous voudrions retourner ici, avec la publication de Peau noire, masques blancs de Frantz Fanon ${ }^{2}$. Il y a dans ce texte, en effet, un concept de relation très bien décrit et qui correspond à cette nuance essentielle que nous proposons ici entre un clivage du sujet et un clivage de la relation. Le texte de Fanon porte sur le racisme, mais dans le racisme, c'est toute la question de l'identité, du corps, du désir et de la relation qui est

1. Toni Morrison, The Bluest Eye, New York, Knopf, 1970.

2. Frantz Fanon, Peau noire masques blancs [1952], Paris, Seuil, coll. "Points", 1971. 
posée. En rejetant toute approche identitaire, en se centrant sur le désir de reconnaissance, et sur le lien entre le désir et le corps, en critiquant fortement l'approche psychanalytique pour son individualisme, Fanon libère la voie à une théorie purement relationnelle du politique qui, nous le verrons, est plus évidente dans ses politiques que dans les concepts qu'il utilise ${ }^{3}$. Il s'agira de prendre le texte moins comme une théorie politique que comme l'écriture d'une voix, d'un désir et d'un projet, dans une relation plurielle entre un "je" et un destinataire appelé.

\section{«Ô mon corps, fais de moi toujours un homme qui interroge »}

L'ouvrage de Fanon, Peau noire, masques blancs, est plus qu'un texte théorique. C'est aussi un texte narratif, presque autobiographique et un manifeste politique, clair et efficace. La voix du texte est donc au moins triple, comme l'est la relation que la lecture impose au lecteur, à tous les lecteurs. Considérons simplement les derniers mots du texte: "Mon ultime prière: Ô mon corps, fais de moi toujours un homme qui interroge!» (p. 188). Le corps est vu comme une instance éthique qui s'impose d'ellemême, aussi bien à l'auteur qu'au lecteur. Car, du point de vue de la lecture, ces derniers mots veulent dire clairement que la voix de celui dont nous avons lu le texte est déterminée indépassablement par la présence matérielle de son propre corps, et donc que cette présence ne saurait être oubliée à aucun moment de la lecture. Pourtant, à aucun moment dans le texte de Fanon, il n'y a de conception essentialiste du corps noir et de la distinction Noir/Blanc qui reste liée à une conception phénoménologique de la présence d'autrui. Le point de vue théorique défendu par Fanon est celui du rapport à autrui, utilisant les concepts sartriens, les concepts hégéliens liés à la reconnaissance de soi, puis les concepts psychanalytiques, notamment l'imaginaire lacanien. Fanon insiste à plusieurs reprises sur le fait que le Noir ne se découvre Noir que face à l'autre, le Blanc. Et, lorsqu'il fait référence

3. Dans l'esprit de Homi K. Bhabha ("Interrogating Identity. Frantz Fanon and the postcolonial prerogative", dans The Location of Culture, Londres \& New York, Routledge, 1994) qui utilise le commentaire de Fanon pour introduire la dimension du désir dans la question postcoloniale de l'identité, nous nous appuierons sur Fanon pour élaborer une théorie de la relation à partir de la façon dont le désir s'inscrit ou non dans les relations du sujet. 
au "réel" afin de s'opposer à toute psychologie individualiste des pathologies liées au racisme, il s'agit toujours de la réalité socioéconomique et historique du colonialisme. Pour lui, ces pathologies sont causées par l'intériorisation de situations politiques contingentes. Il n'y a aucune vérité, nécessité ou essence liée à la négritude. Celle-ci est situationnelle et relationnelle: "la situation coloniale" (p. 76). Cependant, la reconnaissance du Noir en tant qu'homme ne saurait être possible abstraitement. Il ne peut être reconnu en tant qu'homme que dans sa spécificité. Il ne saurait être question d'admettre une reconnaissance désincarnée, hors de la "peau noire". Pour Fanon, il n'y a pas là de contradiction. Car cette spécificité est historique et la reconnaissance exige une transformation du monde, que ce soit celle des conditions socioéconomique et politique, ou celle de l'inconscient collectif. Nous voyons comment les trois modes de Peau noire, masques blancs, les modes narratif, théorique et polémique, sont coreliés. Nous pouvons donner encore en exemple l'humanisme très particulier de Fanon. Il croit en l'homme dans tout ce qu'il a d'universel, ce qui lui permet d'identifier une déshumanisation du Noir et une non-reconnaissance du Noir en tant que conscience de soi. Mais, en même temps, sa théorie ne s'appuie pas sur un humanisme. Elle évoque même des situations qu'un humaniste ne pourrait même pas identifier tant il croit à l'universalité de l'homme. Elle décrit des relations qui sont toujours asymétriques et non réciproques et où nous pouvons concevoir la possibilité d'une nonidentification absolue, d'une altérité non réconciliable: "deux métaphysiques" (p. 6), "deux narcissisme" (p. 7). L'humanisme reste ici un but indéterminé, presque un mouvement révolutionnaire: "Vers un nouvel humanisme..." (p. 5).

Plutôt que de dissocier ces trois modes et d'établir ainsi la cohérence du texte, nous avons choisi de conserver la force de cette écriture, en gardant intacte la tension qui l'anime et de rechercher alors l'unité de la pensée de Fanon dans la conception puissante qu'il se fait de la relation et qui va bien au-delà des références à la psychanalyse et à la philosophie, et à tous les auteurs cités, que ce soit Hegel, Lacan, Sartre ou Jung ${ }^{4}$. Le narratif,

4. Entre bien d'autres côté philosophie et psychanalyse: Alfred Adler, Gaston Bachelard, Marie Bonaparte, Georges Canguilhem, Karl Jaspers, Jean Lacroix, Gabriel Marcel, Maurice Merleau-Ponty, Emmanuel Mounier, Pierre Naville, Jean Wahl, etc.; et côté littérature: Nicolas Calas, David Diop, Léopold 
le politique et le théorique interagissent dans ce concept de relation qui s'élabore au croisement de ces trois modes de discours.

\section{Les relations clivées}

La première dimension de ce concept de relatin qu'il faut relever est celle du clivage (p. 86). Le clivage est décrit de deux façons différentes qui sont pour Fanon indissociables. C'est un clivage du sujet noir colonisé à qui il est imposé comme seul futur et seul désir, celui d'être blanc: "le Noir veut être Blanc" (p. 7). Et c'est en même temps un clivage relationnel qui est identifié à l'aide de la notion hégélienne de reconnaissance. Le Noir n'a pas accès à la lutte pour la reconnaissance, car la condition préalable de toute reconnaissance est d'être Blanc. Il faut déjà être Blanc pour s'inscrire dans la lutte (chapitre 7). Du coup, pour le Noir, le désir d'être Blanc et le désir de reconnaissance sont une seule et même chose. Il en résulte alors un clivage du moi. Or toute l'argumentation de Fanon contre la psychologie individuelle est de soutenir que le clivage du soi n'est que l'effet violent du clivage qui affecte les conditions de la reconnaissance. Et le noud central de la pensée de Fanon se trouve là, dans la façon dont le clivage du moi et le clivage de la relation interagissent.

D'une part, Fanon conserve le langage psychanalytique pour décrire les effets de clivage subis par le sujet, notamment dans la longue note 25 du chapitre 6, "Le Nègre et la psychopathologie". Reprenant les notions lacaniennes de stade du miroir et d'image ${ }^{5}$, Fanon soutient que l'image du Noir telle qu'il se voit lui-même subit une distorsion due au conditions de la reconnaissance de soi dans le regard de l'Autre, l'Autre étant ici l'instance blanche. Les exemples qu'il donne sont frappants:

Nous affirmons que pour les Antillais l'hallucination spéculaire est toujours neutre. À ceux qui disent l'avoir observé chez eux, nous avons posé régulièrement la question: "De quelle couleur étais-tu? - J'étais sans couleur."

Senghor, Paul Valéry, Richard Wright. Aimé Césaire et Jean-Paul Sartre sont cités, faut-il l'ajouter, pour des œuvres qui sont et d'un côté et de l'autre. Enfin, les plus récentes citations, sauf erreur, sont de 1950.

5. Fanon cite l'article de Lacan, "La famille: le complexe, facteur concret de la psychologie familiale. Les complexes familiaux en pathologie", Encyclopédie française, Paris, Larousse, 1938. 
Au sujet posé: "Impression avant de partir en vacances", ils [les enfants antillais] répondent comme de véritables petits parisiens [...] "J'aime les vacances car je pourrai courir à travers les champs, respirer un bon air et je reviendrai avec les joues roses."

L'image de soi du Noir est blanche. C'est ainsi qu'il s'imagine dans le regard de l'Autre, selon une image irréconciliable avec la sienne propre dans le miroir.

D'autre part, tout en reconnaissant ce clivage du moi, Fanon soutient que "le drame racial se déroule en plein air, le Noir n'ayant pas le temps de l'"inconscienciser" (p. 122). Le clivage du moi se joue donc, dans le cas des pathologies noires, dans le réel, au-dehors du soi. Et sur cette position, Fanon ne recule à aucun moment. Les conditions qui ont provoqué le clivage du moi sont pour lui tout à fait contingentes, liées à la situation coloniale. L'enjeu politique est ici considérable. Il s'agit de refuser toute identité noire construite sur la victimisation et de reconnaître la présence d'une violence réelle et symbolique qui brise le sujet, une violence politique qui n'aurait pas lieu d'être et qui aurait pu ne pas être. La question qu'il faut alors se poser est celle de la force symbolique de cette violence. La réponse, à notre avis, ne peut être donnée par les remarques explicites que Fanon fait sur les conditions socio-économique et politique du racisme colonial. Car, entre ces conditions et l'intériorisation de la violence dans le clivage du soi, il y a un hiatus qu'il faut saisir. Nous pouvons bien comprendre comment la lutte pour la modification de ces conditions libère le sujet, en éliminant la cause de la violence subjective, il reste que la force de la violence ne peut s'expliquer que par la nature de la relation que la situation coloniale met en place. Comment se fait-il qu'une situation contingente puisse s'imposer au sujet avec une telle contrainte subjective, s'imposer avec une telle nécessité? Et c'est là que la théorie de Fanon trouve toute sa force, dans la description même qu'il donne des conditions coloniales et racistes de la reconnaissance. Nous voudrions montrer comment Fanon opère le déplacement d'un clivage subjectif à un clivage relationnel. Le Noir qui vit son inconscient au dehors de lui, le vit dans une réalité où les relations sont structurées selon des clivages qui correspondent exactement à ceux qu'il subit dans son image.

Il faut remarquer tout d'abord que la distorsion imposée au sujet colonial dans son désir de reconnaissance est une dimension qui a été, depuis, souvent relevée dans les réflexions sur le colonialisme. Cette restriction selon laquelle le Noir ne peut être 
reconnu comme humain que s'il est d'abord identifié au Blanc se retrouve aussi dans la notion que Jean-François Lyotard propose de la victime dans Le différend ou dans la définition par Gayatri Spivak du subalterne ${ }^{6}$. Dans les deux cas, la violence que le sujet subit est redoublée par son impossibilité à la faire reconnaître, le langage pour le faire étant celui du pouvoir. À un premier niveau, Fanon ne dit pas autre chose.

Pour le Noir, il n'y a qu'un destin. Et il est blanc (p. 8).

Je ne veux pas être reconnu comme Noir, mais comme Blanc (p. 51).

Il faut cependant considérer la description complète qu'il fait de cette relation. Les conditions imposées à la reconnaissance s'accompagnent en même temps d'une non-reconnaissance définitive du corps noir. Il y a dans la relation une réciproque tout à fait asymétrique et déterminante:

Le véritable Autrui du Blanc est et demeure le Noir. Et inversement. Seulement pour le Blanc, Autrui est perçu sur le plan de l'image corporelle, absolument comme le non-moi, c'est-à-dire le non-identifiable, le non-assimilable (p. 131).

À plusieurs reprises, il est mentionné l'exclusion du Noir de l'humain (p. 79). Afin de lier le clivage du moi et le clivage de la relation, il faut donc prendre en compte la totalité des possibilités que le rapport à autrui laisse. Le Noir doit choisir entre être Blanc ou n'être rien, rien d'humain ou de reconnaissable. Cette alternative affecte directement son narcissisme. Il n'a plus le choix quant à son image. Soit il est un corps sans image reconnaissable, du pur "biologique" (p. 134), soit il a une image et elle est "sans couleur" ou modelée sur le désir d'être blanc ("avoir les joues roses"). Dès lors, la force de l'obligation d'être Blanc, de se vouloir Blanc, vient de l'alternative même, de la menace constante de non-existence. C'est ainsi que le Noir vit son clivage au dehors de lui-même. Et il s'agit bien ici, au sein de la relation, d'un clivage, au sens freudien du terme ${ }^{7}$. Les deux termes ainsi clivés ne sont

6. Jean-François Lyotard, Le différend, Paris, Minuit, 1983; Gayatri Spivak, The Spivak Reader. Selected Works of Gayatri Chakravorty Spivak, édition préparée par Donna Landry et Gerald MacLean, New York, Routledge, 1996.

7. Sigmund Freud, "La perte de la réalité dans la névrose et dans la psychose" [1924] et "Névrose et psychose" [1924], dans Névrose, psychose et perversion, Paris, PUF, 1973; "Le clivage du moi dans les processus de défense" [1938], dans Résultats, idées, problèmes, Paris, PUF, 1991. 
aucunement réconciliables: on ne peut être à la fois corps noir et reconnu. En accord avec la théorie psychanalytique, le clivage de la relation suppose bien, par ailleurs, le rejet, la négation, d'une partie de la réalité, à savoir la peau noire elle-même. La nuance qu'il faut cependant faire dans l'identification d'un clivage relationnel est que le retrait d'une partie de la réalité touche d'abord et avant tout les possibilités imaginaires et relationnelles du sujet. Dans l'identification que le sujet noir fait de soi dans son rapport à l'autre, certaines options sont tout simplement éliminées, inaccessibles, même au niveau imaginaire. C'est pourquoi Fanon peut soutenir que le Noir est piégé à la fois dans le "réel" (p. 123) et dans son désir, car il ne peut énoncer son désir que dans les limites de ce qui est "réellement" possible et reconnaissable dans le rapport à autrui.

\section{Désir et relation}

Nous pouvons d'ores et déjà repérer deux caractéristiques de la théorie de la relation mise en place dans Peau noire, masques blancs. Il s'agit d'une relation clivée, indépendamment du sujet qui, au contraire, la subit comme violence symbolique, et d'une relation qui détermine non seulement la réalité, à savoir les conditions contraignantes de la reconnaissance, mais encore les limites du possible, restreignant l'imaginaire lui-même ainsi que le désir. Ce concept de relation va au-delà des analyses théoriques de Fanon lui-même. La référence à la situation politique et économique rend compte du désir de reconnaissance du Noir. L'appel à Jung et à la notion d'inconscient collectif sert à expliquer comment le Noir en vient à être exclu de l'humain, réduit à une pure corporalité sexuelle et biologique (p. 118). Mais la relation dans ce qu'elle a de spécifique, c'est-à-dire dans ce qu'elle a de global, transparaît plutôt dans la position du sujet d'écriture et du Noir comme sujet de désir, face à la relation clivée. Lorsque Fanon pose la question dès les premiers pages de son livre "Que veut l'homme noir? "(p. 6), la question va au-delà de ce que le racisme laisse comme possibilité de désir:

Autrement dit, le Noir ne doit plus se trouver placé devant ce dilemme: se blanchir ou disparaitre, mais il doit pouvoir prendre conscience d'une possibilité d'exister; autrement dit encore, si la société lui fait des difficultés à cause de sa couleur, si je constate dans ses rêves l'expression d'un désir inconscient de changer de couleur, mon but ne sera pas de l'en dissuader en 
104

lui conseillant de "garder ses distances"; mon but, au contraire, sera, une fois les mobiles éclairés, de le mettre en mesure de choisir l'action (ou la passivité) à l'égard de la véritable source conflictuelle — c'est-à-dire à l'égard des structures sociales (p. 80) ${ }^{8}$.

La dimension politique de Peau noire, masques blancs est donc d'identifier les limitations imposées à l'homme noir de façon à l'amener à se poser au-delà, là où il sera un sujet autonome par choix. C'est pourquoi l'humanisme apparent de Fanon est dépassé par sa politique, car il nous montre bien que l'option d'être reconnu à la fois comme noir et comme homme n'est pas encore donnée avec la simple définition de l'homme, mais qu'elle est à produire dans l'agir. Pour le moment, ce qui est donné, c'est un corps dans une position tout à fait problématique et absolument non essentialiste.

Les derniers mots du texte ("Mon ultime prière: Ô mon corps, fais de moi toujours un homme qui interroge!») peuvent désormais recevoir tout leur sens. Le corps du sujet qui écrit, Frantz Fanon, a une position doublement décentrée. D’une part, le clivage de la relation passe à travers lui. Les termes irréconciables du clivage enferment le corps noir dans un lieu impossible, où aucune coïncidence entre le désir et le corps n'est possible. Tout désir d'exister implique une brisure irréparable du soi. D'autre part, le corps noir reste posé au dehors de la relation clivée qui est et reste une contingence politique. Ce corps est aussi un agent qui peut choisir de créer des conditions d'existence au-delà des possibilités imposées. La preuve en est que la relation n'est en rien un destin. Elle ne se met en place et ne devient déterminante que lorsque le Noir se trouve face au Blanc. Fanon insiste à plusieurs reprises sur les modifications de la subjectivité noire lorsqu'un sujet quitte un milieu familial ou social homogène à la rencontre de l'Europe et du monde blanc. Il situe notamment ses analyses par rapport à l'Antillais qui immigre en France, puis revient. L'homme noir, piégé jusque dans son imaginaire et son désir, n'en demeure pas moins un sujet autonome, dans la mesure même où les "structures" qui l'emprisonnent sont contingentes. Mais son autonomie dépend de la conscience qu'il prend de la

8. Henry Louis Gates Jr. ("White like me", The New Yorker, 17 juin 1996) ne dit pas autre chose lorsqu'il analyse politiquement le phénomène du "passing", à savoir celui des Noirs qui passent pour Blancs. 
totalité de ces structures, que nous nous permettons ici de dire relationnelles.

\section{Relations ternaires et plurielles}

Nous voici donc avec trois aspects de la théorie relationnelle de Frantz Fanon: le clivage, la délimitation des identifications possibles et impossibles, le statut du corps désirant qui tout à fois subit le clivage et se pose en agent de son dépassement. Il reste à faire ressortir la structure nécessairement ternaire, et même plurielle, du rapport à Autrui. Il faut entendre par là, non seulement la structure médiatisée de la relation telle que Hegel puis Lacan l'ont pensée autour de l'Autre, de l'esprit ou du symbolique, mais aussi et surtout la présence incontournable et tout à fait concrète du tiers dans toute relation entre le Noir et le Blanc. Le clivage relationnel n'a de sens chez Fanon que si la possibilité des autres est impliquée directement ou indirectement dans le face à face entre moi et l'autre. Si le Blanc impose au Noir d'être ou de désirer être Blanc pour être reconnu, c'est que l'autre pourrait être aussi un Blanc. Il y a, ne l'oublions pas, une indépassable contingence dans toute situation de domination pour Fanon. L'autre peut toujours être Noir ou Blanc, et il n'est pas nécessaire qu'ils se rencontrent. C'est bien pourquoi, lorsqu'une rencontre a lieu, elle peut mettre en place une telle violence du sujet. Cette contingence se marque constamment pas la possible présence des autres en tiers, et de la reconnaissance ou de la non-reconnaissance dont ils feraient l'objet. Cette dernière dimension de la relation est probablement la moins théorisée par Fanon, peut-être même la plus dissimulée dans la pensée jungienne de l'inconscient collectif, mais elle revient constamment dans ses narrations. Nous passons par de nombreuses possibilités de triangle: le Noir face à un Noir parmi les Noirs, le Noir face aux Blancs parmi les Blancs, le Noir face au Noir parmi les Blancs. Et chacune de ces situations contingentes implique une efficience différente du rapport de reconnaissance, selon les effets possibles du clivage, Dans tous les cas, ce qui est mis en évidence par cette structure ternaire si concrète, c'est la contingence de l'identité de chacun qui ne devient politique que par les violences mises en acte ou subies. Ainsi, si le Noir désire être Blanc pour être reconnu par son interlocuteur blanc, c'est qu'il sait que, s'il l'était, la reconnaissance en tant qu'homme lui serait acquise. De même, le corps noir peut bien 
être renvoyé à du non-identifiable, le Blanc pourra toujours trouver un autre homme blanc pour se reconnaître réciproquement comme humain. Ou encore, si les Noirs peuvent se considérer mutuellement comme étant plus ou moins blancs ou noirs, c'est que le troisième, lui, peut toujours être blanc. De façon plus générale, le clivage symbolique prend son sens et son efficacité de cette ségrégation symbolique qui traverse l'ensemble des autres possibles dont le moi et autrui font partie. Il crée du collectif et de l'isolement, définit l'humain et exclut l'inhumain. Ainsi, pour le raciste blanc face à un Noir, les autres en position de tiers sont toujours et nécessairement blancs. Ceux qui ne le sont pas sont déjà exclus d'avance de l'humain. Ou, à l'inverse, pour le Noir qui veut être Blanc, les autres, ceux qui existent en tant que reconnus, ne peuvent être que blancs. Les Noirs sont déjà non identifiables. La distorsion que Fanon impose à la lutte hégélienne pour la reconnaissance n'est possible que parce que la relation est ici ternaire. Les Blancs se reconnaissent entre eux comme hommes qui ont la conscience nécessaire pour reconnâ̂tre le Noir comme libre, lui qui ne saurait le faire seul.

La structure ternaire explique pourquoi le discours politique de Fanon peut faire l'économie de l'identité et pourquoi les différentes positions du corps noir peuvent demeurer des contingences. Le désir du corps noir s'énonce toujours dans le contexte d'une rencontre entre plusieurs, et non dans un face à face. Les relations qui se tissent autour du corps sont donc toujours plurielles. Il faut bien comprendre ici que, s'il en était autrement, il n'y aurait pas de libération possible de la situation coloniale. Pourtant, cette dimension de la relation est la moins explicite dans le texte de Fanon. Celui-ci reste attaché à des modèles plutôt binaires, tels que ceux de Sartre, Hegel, Lacan ou Jung: moi et l'autre, au singulier ou au collectif. Nous répondrons que ces modèles, surtout ceux de Hegel et de Lacan, sont en fait ternaires. Il y a un troisième terme, l'esprit ou Histoire chez le premier, l'Autre et le symbolique chez le second. Mais la place du troisième terme, dans les deux cas, marque la relation, lieu du collectif entendu comme universel et qui rend possible l'ouverture du sujet à l'Autre. Avec Fanon, cette ouverture est atteinte, violemment atteinte, et du coup le tiers est pluriel, la relation pouvant être clivée, ou réinventée. Il suffit pour s'en convaincre de relire Lacan à partir de Fanon, en montrant comment ce dernier remet en question la notion lacanienne d'imaginaire comme espace illusoire du "tout 
est possible" où seul le symbolique, la parole du "je", pourrait déterminer la vérité du sujet. Ici, l'imaginaire est déjà politique, il est déjà déterminé en ce qu'il répartit le possible. Entre moi et l'autre, il y a une instance qui introduit l'asymétrie, mais qui, heureusement l'introduit de plus de façon contingente. Du coup, il y a toujours d'autres voix possibles dans la relation à l'autre. C'est en sens que les relations que Fanon décrit sont plurielles dans leur tierceté 9 .

Seul un tel modèle de relation, ternaire et pluriel, peut rendre compte de la position du sujet de l'écriture dans Peau noire, masques blancs. Le sujet et sa voix supposent que l'autre soit au moins double, raciste, non-Blanc, Noir, ou autre, dans un au-delà de l'opposition. De même, le "je" de Frantz Fanon ne fait sens que s'il est l'autre dans une pluralité de relations possibles. Ainsi, la forme triangulaire de la relation est bien une forme plurielle. Toute relation singulière implique toujours un ensemble de relations divergentes et convergentes ${ }^{10}$. C'est dans ce cadre pluriel que les clivages relationnels prennent toute leur efficacité. Au contraire, le rapport à autrui auquel Fanon fait explicitement référence reste une singularité médiatisée de façon univoque. Pourtant, il tente constamment de briser ce cadre singulier et d'introduire la pluralité de sens. Le reconnaître, c'est reconnaître la voix et l'appel de l'écriture.

\section{Pour une théorie politique de la relation}

L'actualité de Frantz Fanon tiendrait, à notre avis, dans le développement des éléments présents dans son travail pour une

9. La théorie du langage de Lyotard dans Le différend est, à notre avis, ce qui se rapproche le plus d'une pensée de la relation plurielle et ternaire, puisque les phrases sont constituées par des règles hétérogènes et que les genres de discours qui enchaînent les phrases n'ont jamais d'autorité universelle, de telle sorte que certains enchaînements possibles restent inactualisés. Bref, les différends naissent de ce que la tierce instance est toujours contingente, qu'aucune règle de discours n'est universelle. Entre deux interlocuteurs, il n’y a jamais de tiers neutre et absolument légitime. La situation est toujours celle d'une pluralité de relations possibles.

10. Beloved (New York, Knopf, 1987) de Toni Morrison est une démonstration remarquable du pouvoir symbolique des relations plurielles. Morrison construit autour de Beloved, cette enfant revenue de la mort, de la søur vivante et de la mère meurtrière et victime, tout un réseau de relations ternaires où Beloved est identifiée différemment d'un pôle à l'autre. 
théorie politique de la relation. Il ne s'agirait pas d'“une esthétique de la relation" (p. 61) comme le propose Edouard Glissant ${ }^{11}$, mais bien d'une théorie politique. L'esthétique de la relation cherche à penser un mélange des cultures qui éviterait le piège de l'«identité racine unique" (p. 67) et qu'Édouard Glissant entend élaborer à partir des concepts de Deleuze et Guattari, notamment celui de rhizome. Une politique de la relation ne voit pas dans la relation une forme d'ouverture, mais a pour but de redéfinir tous les concepts et situations en termes relationnels, donc aussi bien la violence ou l'oppression que la libération ou la résistance, le sujet pathologique que le sujet qui désire. Elle présente l'intérêt de faire l'économie des notions de culture et d'identité, de la différence entre inconscient individuel et inconscient collectif, puisque la médiation du singulier et du collectif se fait à même la relation qui est toujours singulière dans ses effets réels et collective dans ses possibilités. Les différents éléments que Fanon met en scène dans la force et la beauté de son écriture peuvent servir ici de point de départ d'une telle théorie dont il resterait à fournir l'encadrement conceptuel adéquat. C'est ce que nous avons voulu faire en proposant la notion de relation clivée dans ses liens intimes au clivage de la subjectivité.

11. Édouard Glissant, Introduction à une poétique du divers, Montréal, les Presses de l'Université de Montréal, 1994. 